Publisher: Research Council of Science and Technology, Biratnagar, Nepal p. 53

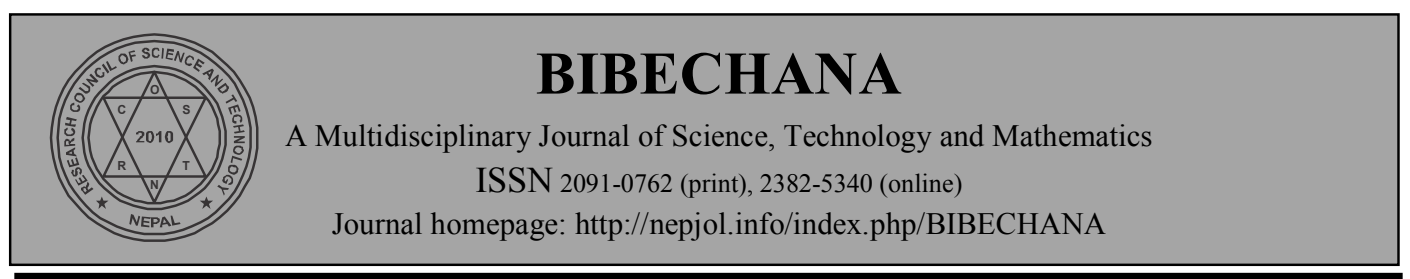

\title{
Energy of Sitnikov's restricted three body problem if the primaries are source of radiation and triaxial rigid bodies
}

\author{
R.R.Thapa ${ }^{1,2^{*}}$ \\ ${ }^{1}$ University Department of Mathematics., T.M. Bhagalpur University, Bhagalpur, India \\ ${ }^{2}$ P.G. Department of Mathematics., P.G. Campus, Biratnagar, T.U., Nepal. \\ *E-mail: thaparajuram@yahoo.com \\ Accepted for publication: December 13, 2014
}

\begin{abstract}
In this paper the joint effect of source of radiation and triaxial rigid body has been studied. The energy of Sitnikov's restricted three body problem when primaries are sources of radiation and energy of Sitnikov's restricted problem of three bodies when primaries are triaxial rigid bodies have been studied to calculate the joint effect. Equation of motion of the third body of infitesimal mass, if primaries are sources of radiation and triaxial rigid bodies, are calculated.
\end{abstract}

DOI: http://dx.doi.org/10.3126/bibechana.v12i0.11707

(C) 2014 RCOST: All rights reserved.

Keywords: Dimensional distance; oscillatory motion; periodicity; radiation parameter.

\section{Introduction}

Sitnikov [1] problem can be integrated in case of restricted problem if primaries are taken to be equal masses. If primaries are triaxial rigid bodies then distance between them will be changed. Then third body can move along $\mathrm{z}$ axis. But if primaries are sources of radiation then the third body moves along the line perpendicular through the centre of mass of the primaries and is perpendicular to the plane of motion.

Thapa et al. [2] obtained the periodicity of Sitnikov's restricted three body problem when the primaries are sources of radiation. They found the equations of motion of the third body and total energy of the system. R.R. Thapa et al. [3] also obtained the equations of motion of the Sitnikov's restricted problem of three bodies when Primaries are triaxial rigid bodies. They got the equations of motion in dimensionless variables and Cartesian form as

$$
\begin{aligned}
\ddot{x}-2 n \dot{y} & =\Omega_{x} \\
\ddot{y}+2 n \dot{x} & =\Omega_{y} \\
\ddot{z} & =\Omega_{z}
\end{aligned}
$$


where $\Omega=\frac{n^{2}}{2}\left(x^{2}+y^{2}\right)+\frac{1-\mu}{r_{1}}+\frac{\mu}{r_{2}}+\frac{(1-\mu)\left(2 \sigma_{1}-\sigma_{2}\right)}{2 r_{1}^{3}}$

$$
\begin{aligned}
& +\frac{\mu\left(2 \sigma_{1}^{\prime}-\sigma_{2}^{\prime}\right)}{2 r_{2}^{3}}-\frac{3(1-\mu)\left(\sigma_{1}-\sigma_{2}\right) y^{2}}{2 r_{1}^{5}} \\
& -\frac{3 \mu\left(\sigma_{1}^{\prime}-\sigma_{2}^{\prime}\right) y^{2}}{2 r_{2}^{5}}-\frac{3(1-\mu) \sigma_{1} z^{2}}{2 r_{1}^{5}}-\frac{3 \mu \sigma_{1}^{\prime} z^{2}}{2 r_{2}^{5}}
\end{aligned}
$$

In present work we have proposed to get the energy of restricted three body problem if the primaries are source of radiation and triaxial rigid bodies.

\section{Equation of motion}

We will adopt the notation and terminology of szehebely [4].

We have the equation of motion of the third body of infinitesimal mass is

$$
\begin{aligned}
& \ddot{z}=\frac{\partial \Omega}{\partial z}=\frac{-(1-p) z}{\left(z^{2}+\frac{a^{2}}{4}\right)^{\frac{3}{2}}}-\frac{3\left(2 \sigma_{1}-\sigma_{2}+2 \sigma_{1}^{\prime}-\sigma_{2}^{\prime}\right) z}{4\left(z^{2}+\frac{a^{2}}{4}\right)^{\frac{5}{2}}}+\frac{15\left(\sigma_{1}+\sigma_{1}^{\prime}\right) z^{3}}{4\left(z^{2}+\frac{a^{2}}{4}\right)^{\frac{7}{2}}} \\
& \frac{d^{2} z}{d t^{2}}=\frac{-(1-p) z}{\left(z^{2}+\frac{a^{2}}{4}\right)^{\frac{3}{2}}}-\frac{3 \alpha z}{4\left(z^{2}+\frac{a^{2}}{4}\right)^{\frac{5}{2}}}+\frac{15}{4} \frac{\left(\sigma_{1}+\sigma_{1}^{\prime}\right) z^{3}}{\left(z^{2}+\frac{a^{2}}{4}\right)^{\frac{7}{2}}}
\end{aligned}
$$

where $\alpha=2 \sigma_{1}-\sigma_{2}+2 \sigma_{1}^{\prime}-\sigma_{2}^{\prime}$

Equation (1) is non-linear equation. It can be integrated by linearizing it.

\section{Energy of the third body}

If we multiply (1) by $2 \frac{d z}{d t}$ we get,

$2 \frac{d z}{d t} \frac{d^{2} z}{d t^{2}}=-\frac{2(1-p) z}{\left(z^{2}+\frac{a^{2}}{4}\right)^{\frac{3}{2}}} \frac{d z}{d t}-\frac{3 \alpha}{4\left(z^{2}+\frac{a^{2}}{4}\right)^{\frac{5}{2}}} \times 2 z \frac{d z}{d t}+\frac{15\left(\sigma_{1}+\sigma_{1}^{\prime}\right) z^{3}}{4\left(z^{2}+\frac{a^{2}}{4}\right)^{\frac{7}{2}}} \frac{2 d z}{d t}$

Or, $\quad \frac{1}{2} \frac{d}{d t}\left(\frac{d z}{d t}\right)^{2}=-\frac{(1-p) z}{\left(z^{2}+\frac{a^{2}}{4}\right)^{\frac{3}{2}}} \frac{d z}{d t}-\frac{3 \alpha z}{4\left(z^{2}+\frac{a^{2}}{4}\right)^{\frac{5}{2}}} \times \frac{d z}{d t}+\frac{15\left(\sigma_{1}+\sigma_{1}^{\prime}\right)}{4} \frac{\left(z^{2}+\frac{a^{2}}{4}-\frac{a^{2}}{4}\right) z}{\left(z^{2}+\frac{a^{2}}{4}\right)^{\frac{7}{2}}} \frac{d z}{d t}$ 
Thapa/ BIBECHANA 12 (2015) 53-58: p. 55

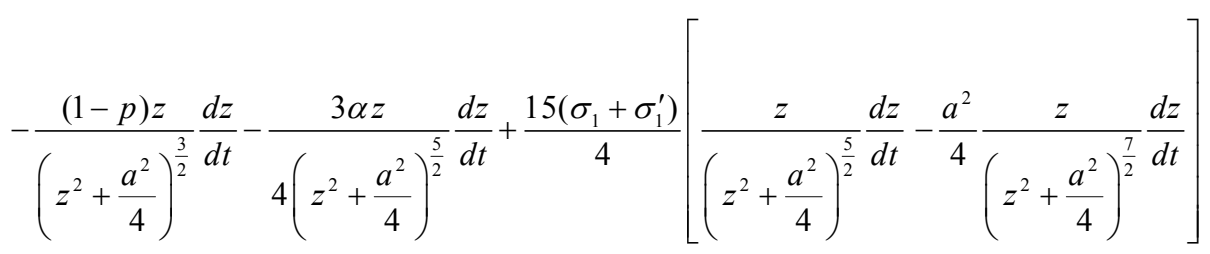

If we substitute $u \frac{d u}{d t}=z \frac{d z}{d t}$ and $u^{2}=z^{2}+\frac{a^{2}}{4}$ we get,

$$
\begin{aligned}
& \frac{1}{2} \frac{d}{d t}\left(\frac{d z}{d t}\right)^{2}=-\frac{(1-p)}{\left(u^{2}\right)^{\frac{3}{2}}} u \frac{d u}{d t}-\frac{3 \alpha}{4\left(u^{2}\right)^{\frac{5}{2}}} u \frac{d u}{d t}+\frac{15\left(\sigma_{1}+\sigma_{1}^{\prime}\right)}{4}\left[\frac{1}{\left(u^{2}\right)^{\frac{5}{2}}} u \frac{d u}{d t}-\frac{a^{2}}{4} \frac{1}{\left(u^{2}\right)^{\frac{7}{2}}} u \frac{d u}{d t}\right] \\
& =-\frac{(1-p)}{u^{2}} \frac{d u}{d t}-\frac{3 \alpha}{4 u^{4}} \frac{d u}{d t}+\frac{15\left(\sigma_{1}+\sigma_{1}^{\prime}\right)}{4}\left[\frac{1}{u^{4}} \frac{d u}{d t}-\frac{1}{4} a^{2} \frac{1}{u^{6}} \frac{d u}{d t}\right] \\
& =-\frac{1}{u^{2}} \frac{d u}{d t}-\frac{3 \alpha}{4 u^{4}} \frac{d u}{d t}+\frac{15}{4}\left(\sigma_{1}+\sigma_{1}^{\prime}\right) \frac{1}{u^{4}} \frac{d u}{d t}-\frac{15}{4}\left(\sigma_{1}+\sigma_{1}^{\prime}\right) \frac{a^{2}}{4} \cdot \frac{1}{u^{6}} \frac{d u}{d t} \\
& =\frac{(1-p) d u^{-1}}{d t}+\frac{\alpha}{4} \frac{d u^{-3}}{d t}-\frac{5\left(\sigma_{1}+\sigma_{1}^{\prime}\right)}{4} \frac{d u^{-3}}{d t}+\frac{3\left(\sigma_{1}+\sigma_{1}^{\prime}\right) a^{2}}{16} \frac{d}{d t}\left(u^{-5}\right) \\
& \frac{1}{2} \int \frac{d}{d t}\left(\frac{d z}{d t}\right)^{2} d t=(1-p) \int \frac{d u^{-1}}{d t} d t+\frac{\alpha}{4} \int \frac{d u^{-3}}{d t} d t-\frac{5\left(\sigma_{1}+\sigma_{1}^{\prime}\right)}{4} \int \frac{d u^{-3}}{d t} d t \frac{3\left(\sigma_{1}+\sigma_{1}^{\prime}\right) a^{2}}{16} \int \frac{d u^{-5}}{d t} d t \\
& \Rightarrow \frac{1}{2}\left(\frac{d z}{d t}\right)^{2}=(1-p) u^{-1}+\frac{\alpha}{4} u^{-3}-\frac{5}{4}\left(\sigma_{1}+\sigma_{1}^{\prime}\right) u^{-3}+\frac{3\left(\sigma_{1}+\sigma_{1}^{\prime}\right) a^{2}}{16} u^{-5}+c \\
& \Rightarrow \frac{1}{2}\left(\frac{d z}{d t}\right)^{2}=\frac{(1-p)}{u}+\frac{\alpha}{4 u^{3}}-\frac{5\left(\sigma_{1}+\sigma_{1}^{\prime}\right)}{4 u^{3}}+\frac{3\left(\sigma_{1}+\sigma_{1}^{\prime}\right) a^{2}}{16} \frac{1}{u^{5}}+c \\
& \Rightarrow \frac{1}{2}\left(\frac{d z}{d t}\right)^{2}=\frac{(1-p)}{\sqrt{z^{2}+\frac{a^{2}}{4}}}+\frac{\alpha}{4\left(z^{2}+\frac{a^{2}}{4}\right)^{\frac{3}{2}}}-\frac{5\left(\sigma_{1}+\sigma_{1}^{\prime}\right)}{4\left(z^{2}+\frac{a^{2}}{4}\right)^{\frac{3}{2}}}+\frac{3\left(\sigma_{1}+\sigma_{1}^{\prime}\right) a^{2}}{16\left(z^{2}+\frac{a^{2}}{4}\right)^{\frac{5}{2}}}+c \\
& \Rightarrow \frac{1}{2}\left(\frac{d z}{d t}\right)^{2}-\frac{(1-p)}{\sqrt{z^{2}+\frac{a^{2}}{4}}}-\frac{\alpha}{4\left(z^{2}+\frac{a^{2}}{4}\right)^{\frac{3}{2}}}+\frac{5\left(\sigma_{1}+\sigma_{1}^{\prime}\right)}{4\left(z^{2}+\frac{a^{2}}{4}\right)^{\frac{3}{2}}}-\frac{3\left(\sigma_{1}+\sigma_{1}^{\prime}\right) a^{2}}{16\left(z^{2}+\frac{a^{2}}{4}\right)^{\frac{5}{2}}}=c \\
& \Rightarrow \frac{1}{2}\left(\frac{d z}{d t}\right)^{2}-\frac{(1-p)}{\sqrt{z^{2}+\frac{a^{2}}{4}}}+\frac{\left(5 \sigma_{1}+5 \sigma_{1}^{\prime}-2 \sigma_{1}+\sigma_{2}-2 \sigma_{1}^{\prime}+\sigma_{2}^{\prime}\right)}{4\left(z^{2}+\frac{a^{2}}{4}\right)^{\frac{3}{2}}}-\frac{3\left(\sigma_{1}+\sigma_{1}^{\prime}\right) a^{2}}{16\left(z^{2}+\frac{a^{2}}{4}\right)^{\frac{5}{2}}}=c
\end{aligned}
$$




$$
\begin{aligned}
& \Rightarrow \frac{1}{2}\left(\frac{d z}{d t}\right)^{2}-\frac{(1-p)}{\sqrt{z^{2}+\frac{a^{2}}{4}}}+\frac{\left(3 \sigma_{1}+\sigma_{2}+3 \sigma_{1}^{\prime}+\sigma_{2}^{\prime}\right)}{4\left(z^{2}+\frac{a^{2}}{4}\right)^{\frac{3}{2}}}-\frac{3\left(\sigma_{1}+\sigma_{1}^{\prime}\right) a^{2}}{16\left(z^{2}+\frac{a^{2}}{4}\right)^{\frac{5}{2}}}=c \\
& \Rightarrow \frac{1}{2}\left(\frac{d z}{d t}\right)^{2}-\frac{(1-p)}{\left(z^{2}+\frac{a^{2}}{4}\right)^{\frac{1}{2}}}+\frac{p}{4\left(z^{2}+\frac{a^{2}}{4}\right)^{\frac{3}{2}}}-\frac{3\left(\sigma_{1}+\sigma_{1}^{\prime}\right) a^{2}}{16\left(z^{2}+\frac{a^{2}}{4}\right)^{\frac{5}{2}}}=\text { constant }
\end{aligned}
$$

$$
E=\frac{1}{2}\left(\frac{d z}{d t}\right)^{2}-\frac{(1-p)}{\left(z^{2}+\frac{a^{2}}{4}\right)^{\frac{1}{2}}}+\frac{p}{4\left(z^{2}+\frac{a^{2}}{4}\right)^{\frac{3}{2}}}-\frac{3\left(\sigma_{1}+\sigma_{1}^{\prime}\right) a^{2}}{16\left(z^{2}+\frac{a^{2}}{4}\right)^{\frac{5}{2}}}=\text { constant }
$$

$$
E=\frac{1}{2}\left(\frac{d z}{d t}\right)^{2}-\frac{(1-p)}{\sqrt{z^{2}+\frac{a^{2}}{4}}}+\frac{p}{4\left(z^{2}+\frac{a^{2}}{4}\right)^{\frac{3}{2}}}-\frac{3\left(\sigma_{1}+\sigma_{1}^{\prime}\right)}{16\left(z^{2}+\frac{a^{2}}{4}\right)^{\frac{5}{2}}}=\text { constant. }
$$

Now from equation (1)

$$
\begin{gathered}
\quad \frac{d^{2} z}{d t^{2}}+\frac{(1-p) z}{\left(z^{2}+\frac{a^{2}}{4}\right)^{\frac{3}{2}}}+\frac{3 \alpha z}{4\left(z^{2}+\frac{a^{2}}{4}\right)^{\frac{5}{2}}}-\frac{15\left(\sigma_{1}+\sigma_{1}^{\prime}\right)}{4\left(z^{2}+\frac{a^{2}}{4}\right)^{\frac{7}{2}}} z^{3}=0 \\
\Rightarrow \frac{d^{2} z}{d t^{2}}+\frac{(1-p) z}{\left(\frac{a}{2}\right)^{3}\left(1+\frac{4 z^{2}}{a^{2}}\right)^{\frac{3}{2}}}+\frac{3 \alpha z}{4\left(\frac{a}{2}\right)^{5}\left(1+\frac{4 z^{2}}{a^{2}}\right)^{\frac{5}{2}}}-\frac{15\left(\sigma_{1}+\sigma_{1}^{\prime}\right)}{4\left(\frac{a}{2}\right)^{7}\left(1+\frac{4 z^{2}}{a^{2}}\right)^{\frac{7}{2}}} z^{3}=0 \\
\Rightarrow \frac{d^{2} z}{d t^{2}}+\frac{(1-p) 8 z}{a^{3}}\left(1+\frac{4 z^{2}}{a^{2}}\right)^{-\frac{3}{2}}+\frac{24 \alpha z}{a^{5}}\left(1+\frac{4 z^{2}}{a^{2}}\right)^{-\frac{5}{2}} \quad\left(\text { as } z<<a \quad \text { so }\left(\frac{z}{a}\right)^{<<<1)}\right. \\
\left.-\frac{15\left(\sigma_{1}+\sigma_{1}^{\prime}\right) \times 32 z^{3}}{a^{7}}\left(1+\frac{4 z^{2}}{a^{2}}\right)^{-\frac{7}{2}}=0 \quad \frac{7}{a^{2}}(2 z)^{2}+\ldots\right)=0 \\
\Rightarrow \frac{d^{2} z}{d t^{2}}+\frac{(1-p) 8 z}{a^{3}}\left(1-\frac{3}{2}\left(\frac{2 z}{a}\right)^{2}+\ldots\right)+\frac{24 \alpha z}{a^{5}}\left(1-\frac{5}{2}\left(\frac{2 z}{a}\right)^{2}+\ldots\right)-\frac{15 \times 32}{a^{7}}\left(\sigma_{1}+\sigma_{1}^{\prime}\right) z^{3}\left(1-\frac{7}{2}\left(\frac{2}{a}\right)^{2}\right)
\end{gathered}
$$

Neglecting higher order term of $(\mathrm{z} / \mathrm{a})$ above the third order, we get, 


$$
\begin{aligned}
& \frac{d^{2} z}{d t^{2}}+\frac{(1-p) 8 z}{a^{3}}-\frac{8 z}{a^{3}} \cdot \frac{12}{2}\left(\frac{z}{a}\right)^{2}+\frac{24 \alpha z}{a^{5}}-\frac{24 \alpha z}{a^{5}} \times \frac{5 \times 4 z^{2}}{2 a^{2}}-\frac{480\left(\sigma_{1}+\sigma_{1}^{\prime}\right)}{a^{7}} z^{3} \\
& \quad+\frac{480\left(\sigma_{1}+\sigma_{1}^{\prime}\right)}{a^{7}} z^{3} \frac{7}{2} \times \frac{4 z^{2}}{a^{2}}=0 \\
& \frac{d^{2} z}{d t^{2}}+\left(\frac{(1-p) 8}{a^{3}}+\frac{24 \alpha}{a^{5}}\right) z-\left(\frac{48}{a^{5}}+\frac{240 \alpha}{a^{7}}+\frac{480\left(\sigma_{1}+\sigma_{1}^{\prime}\right)}{a^{7}}\right) z^{3}+\ldots=0 \\
& \frac{d^{2} z}{d t^{2}}+\frac{8(1-p)}{a^{5}}\left(a^{2}+3 \alpha\right) z-\frac{48}{a^{7}}\left(a^{2}+5 \alpha+10\left(\sigma_{1}+\sigma_{1}^{\prime}\right)\right) z^{3}=0 \\
& \frac{d^{2} z}{d t}+\eta_{0}^{2} z-\varepsilon z^{3}=0 \\
& \text { where } \eta_{0}^{2}=\frac{8(1-p)}{a^{5}}\left(a^{2}+3 \alpha\right)=\frac{8 q\left(a^{2}+3 \alpha\right)}{a^{5}} \\
& \text { and } \quad \varepsilon=\frac{48}{a^{7}}\left[a^{2}+5 \alpha+10\left(\sigma_{1}+\sigma_{1}^{\prime}\right)\right]
\end{aligned}
$$

The equations (3), (4) represent energy of Sitnikov's restricted three body problem if the primaries are source of radiation and triaxial rigid bodies.

\section{Result and Discussion}

The energy of third body depends on variable $\mathrm{z}$, radiation parameter $\mathrm{p}$, (parameter) $\alpha$ $=2 \sigma_{1}-\sigma_{2}+2 \sigma_{1}{ }^{1}-\sigma_{2}{ }^{1}, \quad$ distance between the primaries $a$; where $\sigma_{1}=\frac{\mathrm{a}_{1}{ }^{2}-\mathrm{a}_{3}{ }^{2}}{5 \mathrm{R}^{2}}$,

$$
\sigma_{2}=\frac{a_{2}{ }^{2}-a_{3}{ }^{2}}{5 R^{2}}, \sigma_{1}^{2}=\frac{b_{1}{ }^{2}-b_{3}{ }^{2}}{5 R^{2}}, \sigma_{2}{ }^{1}=\frac{b_{2}{ }^{2}-b_{3}{ }^{2}}{5 R^{2}} ; R \text { is dimensional distance between the }
$$

primaries and $a_{1}, a_{2}, a_{3} ; b_{1}, b_{2}, b_{3}$ are lengths of semi-axes of two primaries parallel to axes respectively. The kinetic energy of the third body depends on variable $\mathrm{z}$ but potential energy depends on $\eta_{0}$ and $\varepsilon$.

where $\eta_{0}^{2}=8 \mathrm{q} \frac{\left(\mathrm{a}^{2}+3 \alpha\right)}{\mathrm{a}^{5}}$ and $\varepsilon=\frac{48}{\mathrm{a}^{7}}\left[\left(\mathrm{a}^{2}+5 \alpha+10\left(\sigma+\sigma^{1}\right)\right]\right.$.

Hence the third body will perform oscillatory motion.

\section{Acknowledgement}

I want to acknowledge with thanks my supervisor Dr. M.R. Hassan, Department of Mathematics, S.M. College, Bhagalpur, T.M.B.U. Bghagalpur, 812007, India for his meticulous guidance during the work. 


\section{References}

[1] K.A. Sitnikov, Dokl. Akad, Nauk, USSR 132(2) (1960) 303.

[2] R.R. Thapa, and M.R. Hassan, Journal of the Bihar Mathematical society 27(2013) 99105.

[3] R.R. Thapa and M.R. Hassan, A research journal in mathematics " $\mathrm{M}_{0}$ " 1(2014) 37-39.

[4] V. Szebehely, Theory of orbits, Academic Press, New York (1967). 